\title{
On sensitivity in transfer impedance measurements
}

\author{
Fred J. Pettersen ${ }^{1,2,3}$ \\ 1. Department of Clinical and Biomedical Engineering, Oslo University Hospital HF, Oslo, Norway \\ 2. Department of Physics, University of Oslo, Oslo, Norway \\ 2. E-mail any correspondence to: frepet@ous-hf.no
}

\begin{abstract}
The term sensitivity is sometimes misused when discussing volume impedance measurements. This is a critique of the name of the quantity sensitivity, as well as pointing out how the term easily can be misinterpreted. To resolve the issue, a shift of focus towards volume impedance density, which is a more useful quantity, is proposed. A new parameter, perceptivity, is introduced. Perceptivity is useful tool for characterization of measurement systems, to objectively compare systems, and to formulate instrument specifications.
\end{abstract}

Keywords: Bioimpedance; perceptivity; sensitivity

\section{Introduction}

There are a number of parameters that are used to describe properties of a measurement system which consist of measurement instrumentation and measurand. One of these parameters has been given the name sensitivity, which may be misleading. The word sensitivity can be interpreted in at least two ways for a measurement system. Firstly, it specifies how much the output of a system, for example a transducer, changes as a function of a change in the input. This is the classical engineering point-of-view. When considering a 3D volume impedance system, such as a bioimpedance system, sensitivity takes another meaning as explained below.

A short repetition of some of the mathematics involved is useful in order to explain the problem. For those requiring more detailed information on the subject, the book Bioimpedance and Bioelectricity Basics [1] is recommended. If we look at an impedance measurement system with separated current carrying (CC) electrode pair and potential pick-up (PU) electrode pair, the measured transfer impedance is the potential on the PU electrodes divided by the current flowing in the $\mathrm{CC}$ electrode pair. To simplify the text, we are using the term impedance instead of the more proper term transfer impedance. The resulting current density of every point throughout the measurand can be quantified by vectors $\overrightarrow{J_{C C}}$ where $\mathrm{CC}$ reflects that this is the current density of the $\mathrm{CC}$ electrodes. The principle of reciprocity states that we can swap the $\mathrm{CC}$ and $\mathrm{PU}$ electrode pairs [2]. This would result in current density vectors $\overrightarrow{J_{P U}}$ throughout the measurand. We can use the current vector field to calculate what we (currently) call sensitivity as shown in equation 1 ,

$$
S=\frac{\overrightarrow{J_{C C}} \cdot \overrightarrow{J_{P U}}}{I_{C C} I_{P U}}
$$

where $S$ is sensitivity in all points throughout the measurand, $I_{C C}$ is current in CC electrodes, and $I_{P U}$ is the reciprocal current in the PU electrodes [1].

If the sensitivity is multiplied with the resistivity $(\rho)$ as shown as shown in equation 2 we get the volume impedance density $(\mathbf{z})$.

$$
\boldsymbol{z}=S \boldsymbol{\rho}
$$

The volume impedance density can be integrated over all points in the measurand to get the measured impedance, $Z$, as shown in equation 3 .

$$
\boldsymbol{Z}=\iiint \mathbf{z} d V
$$

From equations 2 and 3, we see that both sensitivity and resistivity are required quantities when determining measured impedance which is the core of the critique: It is easy to be misled to believe that sensitivity as defined in equation 1 is telling everything there is to know about the sensitivity in a given point of the measurand. The sensitivity is giving important information, especially about which sign 
of the contribution of a given point, which can be negative if the two current density vectors have an angle of more than $90^{\circ}$, but the contribution to the measured impedance is also requiring knowledge of resistivity. Equation 2 defines volume impedance density, which is including both sensitivity and resistivity, which makes this parameter much more interesting since it tells us how much a point of a measurand is actually contributing to the measured impedance.

But still, the volume impedance density is only giving information of points of the measurand, and we still need a parameter that tell us something about how much measured impedance changes as a result of changes in the measurand. It must be mentioned that David B. Geselowitz touched the subject of reciprocity and sensitivity as defined later as perceptivity in 1971 [3].

Materials and methods

\section{Perceptivity}

In engineering, the word sensitivity refers to how much the output of a system, typically a transducer, changes per change in input. This definition is only weakly related to the quantity defined in section 1 , which is a problem since the engineering definition is what we actually need when evaluating a measurement system. To get around this problem, we use a synonym of the word sensitivity, perceptivity, to which we attach the engineering definition of sensitivity. Described in mathematical terms as

$$
\boldsymbol{\Psi}=\frac{\delta \boldsymbol{Z}}{\delta p}
$$

where $\Psi$ is perceptivity, $\delta \boldsymbol{Z}$ is change in measured impedance, and $\delta p$ is change in the phenomenon we want to test perceptivity against. Since an impedance change is dependent on both geometry and material (tissue) properties, we may specify this by presenting $\Psi_{g}$ and $\Psi_{m}$, respectively. Note that perceptivity as defined in equation 4 is a complex quantity as opposed to the world of engineering, where sensitivity normally is a scalar quantity. From a mathematical point of view, this is no problem, and the perceptivity could even be expanded to be a function of frequency $(\Psi(f))$, but for many cases the simple scalar number is desired. Reduction of a complex, or even frequency dependent, perceptivity to a scalar can be done in many ways as long as the method is specified. Examples are use of real or imaginary values, or magnitude of the $\Psi$ vector. Unit of perception is not mentioned since the phenomenon of interest may vary.

Since a measured quantity often vary around an offset value, it can be useful to expand the perceptivity concept to what we call relative perceptivity defined as

$$
\Psi_{r}=\frac{\frac{\delta Z}{\delta p}}{Z_{0}}=\frac{\Psi}{Z_{0}}
$$

where $\Psi_{r}$ is relative perceptivity and $Z_{0}$ is the starting offset (or bias) a given impedance is varying around. In relative perceptivity, the unit of impedance is taken out of the equation, and if the phenomena have no unit, the perceptivity is unit-less.

\section{Finite Element Model Examples}

Three examples are modelled in the Finite Element Model (FEM) tool COMSOL Multiphysics. For simplicity, the models are for stationary conditions. Only the three different material types shown in table 1 are used. The equations defining the model behavior is predefined in the COMSOL Multiphysics physics interface electric currents.

Tab.1: Electrical properties of the materials used in FEM simulations.

\begin{tabular}{lcc}
\hline \multicolumn{1}{c}{ Material } & Conductivity $(\mathbf{S} / \mathbf{m})$ & Resistivity $(\mathbf{\Omega m})$ \\
\hline lowConductivity & 0.1 & 10 \\
mediumConductivity & 1 & 1 \\
highConductivity & 10 & 0.1 \\
\hline
\end{tabular}

Everything except the electrode placements are identical in the three models. This let us investigate three electrode placements with focus on sensitivity, volume impedance density, and perceptivity. Model figures are shown in figures 1 where current density vector colors can be used to identify CC and PU electrodes. Model A has sideby-side CC and PU electrodes placed far from the center region, model $\mathrm{B}$ has in-line $\mathrm{CC}$ and $\mathrm{PU}$ electrodes placed far from the center region, while model $C$ has in-line $C C$ and $P U$ electrodes with CC electrodes placed far from the center region and $\mathrm{PU}$ electrodes placed near the center region. Four variants of the models are simulated:

1. Base model.

2. $10 \%$ increase of length of center section.

3. $10 \%$ increase of conductivity of center section.

4. $10 \%$ increase of both length and conductivity of center section.

\section{Ethical approval}

The conducted research is not related to either human or animal use.

\section{Results}

The simulation results are shown in figures 1 through 3, and in table 1.

\section{Discussion}

The current density vectors shown in figure 1 is the basis for calculating the sensitivity fields for the models as shown in figure 2 . The sensitivity field for the center piece is more or less the same as it is for the surrounding regions for all models. This is misleading because the contribution from the center piece is much higher than it is for the surrounding regions. Figure 3 shows the volume impedance density field which gives information on how much each 
point contributes to the final measured (or simulated) impedance. The sensitivity field plots have correct sign, as do the volume impedance density field plots, but there stops the usability of sensitivity plots for this particular example.
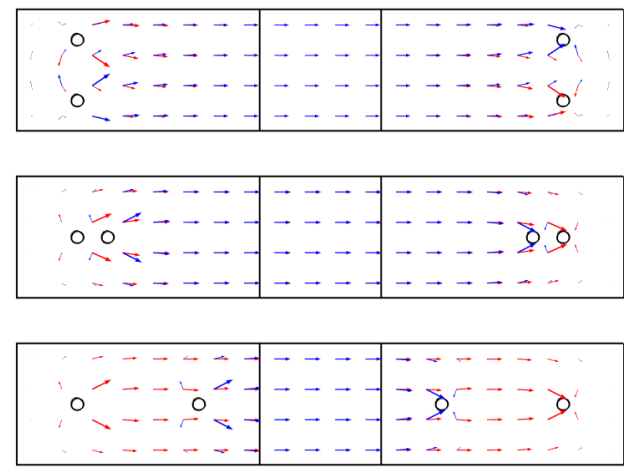

Fig.1: Current density vector plots for the three models, starting with model $A$ at the top followed by model $B$ and $C$. The red vectors are CC current densities and the blue vectors are reciprocal current densities.
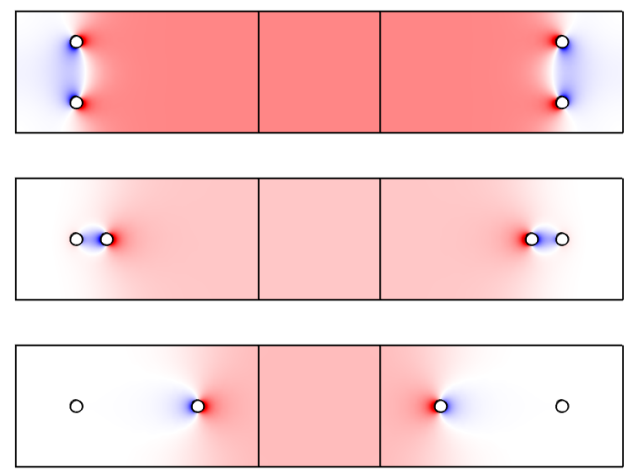

Fig.2: Sensitivity field plots for the three models, starting with model $A$ at the top followed by model $B$ and $C$. The red regions represent positive sensitivity while the blue regions represent negative sensitivity. The absolute value is higher the stronger the color is.
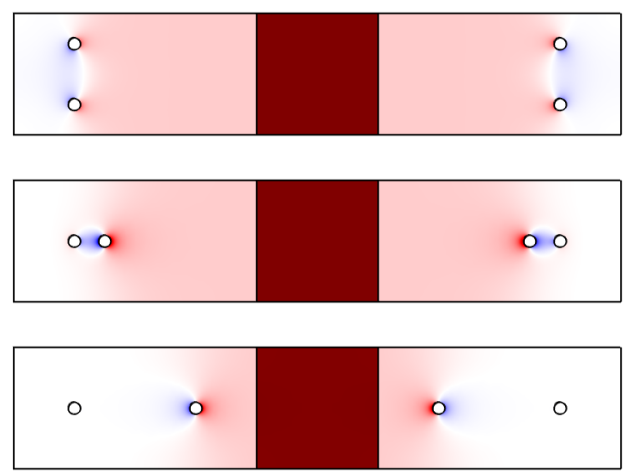

Fig.3: Volume impedance density field plots for the three models, starting with model $A$ at the top followed by model $B$ and $C$. The red regions represent positive volume impedance density while the blue regions represent negative volume impedance density. The absolute value is higher the stronger the color is.
Tab. 2: Simulated impedances and associated perceptivities for the three models. Units for perceptivities are left out since we are considering change in phenomena which can be non-physical changes.

\begin{tabular}{cccc}
\hline Parameter & Model A & Model B & Model C \\
\hline Sim. 1 & $12.645 \Omega$ & $12.645 \Omega$ & $10.985 \Omega$ \\
Sim. 2 & $11.736 \Omega$ & $11.736 \Omega$ & $10.076 \Omega$ \\
Sim. 3 & $13.545 \Omega$ & $13.545 \Omega$ & $11.885 \Omega$ \\
Sim. 4 & $12.545 \Omega$ & $12.545 \Omega$ & $10.885 \Omega$ \\
$\Psi_{\mathrm{m}}$ & -3.636 & -3.636 & -3.636 \\
$\Psi_{\mathrm{g}}$ & 3.600 & 3.600 & 3.600 \\
$\Psi$ & -0.400 & -0.400 & -0.400 \\
$\Psi_{\mathrm{rm}}$ & -0.288 & -0.288 & -0.331 \\
$\Psi_{\mathrm{rg}}$ & 0.266 & 0.266 & 0.303 \\
$\Psi_{\mathrm{r}}$ & -0.032 & -0.032 & -0.036 \\
\hline
\end{tabular}

In cases where the resistivity is constant throughout the model such as the model presented by Brown et. al. and Høyum et.al. [4, 5], the sensitivity field will give the similar information as the volume impedance density field. This is particularly true for graphical visualizations, but the sensitivity cannot be used to determine measured impedance by integration. So even for these cases, the volume impedance field is more useful.

From the volume impedance density field plots in figure 3, it is clear that all models have high $\boldsymbol{z}$ in center piece, and that there are some differences when it comes to the surrounding regions. One can see that model $\mathrm{C}$ is more focused on the center piece, but we have to look at the perceptivities to verify this. Table 2 shows that the perceptivities for all models are identical when using only three decimal places. This means that the change in impedance is the same for each model, which makes sense since the changes happens in the center piece with highest z. If we look at the relative perceptivities, on the other hand, these show that models A and B are very similar, and that model $C$ has about $14 \%$ higher value, which means that this electrode configuration is superior to the ones in model $A$ and $B$. We have thus a tool that enables us to objectively compare electrode configurations.

The perceptivities is also useful to estimate requirements for the instrumentation. If, for example, we inspect the results for model $\mathrm{C}$ in table 2 , we see that the perceptivity for geometrical changes is 3.6. This means that the maximum impedance will change by $3.6 \Omega$. So if we want to distinguish between $n$ different levels, the accuracy must be better than $3.6 / n \Omega$.

\section{Conclusion}

I have shown that volume impedance density is more useful to plot than sensitivity. A sensitivity plot may give a misleading impression of the contributions, and should thus be avoided. Calculation of relative perceptivities gives us tool to objectively compare how good a measurement setup is. It also let gives information regarding requirements for the impedance measurement instrumentation. 


\section{Conflict of interest}

Author declares no conflict of interest.

\section{References}

1. Grimnes, S. and $\varnothing$.G. Martinsen, Bioimpedance and Bioelectricity Basics. 3 ed. 2015: Elsevier Science.

2. Helmholtz, H.v., Ueber einige Gesetze der Vertheilung elektrischer Ströme in körperlichen Leitern, mit Anwendung auf die thierisch-elektrischen Versuche. Annalen der Physik und Chemie 89, 1853.
3. Geselowitz, D.B., An application of electrocardiographic lead theory to impedance plethysmography. IEEE transactions on bio-medical engineering, 1971. 18: p. 38-41. https://doi.org/10.1109/TBME.1971.4502787

4. Brown, B.H., A.J. Wilson, and P. Bertemes-Filho, Bipolar and tetrapolar transfer impedance measurements from volume conductor. Electronics Letters, 2000. 36(25): p. 2060-2060. https://doi.org/10.1049/el:20001439

5. Høyum, P., et al., Finite element model of needle electrode sensitivity. Journal of Physics: Conference Series, 2010. 224(1): p. 012139.

https://doi.org/10.1088/1742-6596/224/1/012139 